\title{
Impact of Ixekizumab Treatment on Depressive Symptoms and Systemic Inflammation in Patients with Moderate-to-Severe Psoriasis: An Integrated Analysis of Three Phase 3 Clinical Studies
}

\author{
Christopher E.M. Griffiths ${ }^{a} \quad$ Maurizio Favab Andrew H. Miller ${ }^{c} \quad$ James Russelld, e \\ Susan G. Ball ${ }^{\mathrm{E}}$ Wen Xu ${ }^{\mathrm{e}}$ Nayan Acharya ${ }^{\mathrm{e}}$ Mark Hyman Rapaport ${ }^{\mathrm{c}}$ \\ a Dermatology Centre, University of Manchester, Manchester Academic Health Science Centre, Manchester, UK; \\ ${ }^{b}$ Department of Psychiatry, Massachusetts General Hospital, Boston, MA, 'Department of Psychiatry and Behavioral \\ Sciences, Emory University School of Medicine, Atlanta, GA, ${ }^{d}$ Department of Psychiatry, Oregon Health Sciences \\ University, Portland, OR, and ${ }^{\mathrm{E}}$ Eli Lilly and Company, Indianapolis, IN, USA
}

\section{Keywords}

Ixekizumab · Psoriasis · Depressive symptoms ·

High-sensitivity C-reactive protein - Comorbid depression . UNCOVER

\begin{abstract}
Background: Depression is a common comorbidity in psoriasis, and both conditions are associated with systemic inflammation. The efficacy of ixekizumab, a high-affinity monoclonal antibody that selectively targets interleukin (IL)17A, was evaluated in patients with moderate-to-severe plaque psoriasis (psoriasis) and depressive symptoms that were at least moderately severe. Methods: Data were integrated from 3 randomized, double-blind, controlled phase 3 trials. At baseline and week 12, depressive symptoms and inflammation were assessed by the 16-item Quick Inventory of Depressive Symptomology - Self-Report (QIDS-SR ${ }_{16}$ ) and by a high-sensitivity assay of serum C-reactive protein (hsCRP), respectively. A subgroup of patients with at least moderately severe depressive symptoms at baseline (QIDS$\mathrm{SR}_{16}$ total score $\geq 11$ ) was analyzed. Improvement in psoriasis
\end{abstract}

\section{KARGER}

E-Mail karger@karger.com www.karger.com/pps

\section{Karger}

Open access

This article is licensed under the Creative Commons Attribution NonCommercial-NoDerivatives 4.0 International License (CC BYNC-ND) (http://www.karger.com/Services/OpenAccessLicense) Usage and distribution for commercial purposes as well as any distribution of modified material requires written permission. was assessed by the Psoriasis Area and Severity Index (PASI). Results: Approximately $10 \%$ of the overall psoriasis population had at least moderately severe depressive symptoms at baseline. At week 12, comorbid patients treated with ixekizumab had significantly greater improvements in their QIDS$\mathrm{SR}_{16}$ total score (ixekizumab $80 \mathrm{mg}$ every 2 weeks [Q2W], -7.1 ; ixekizumab $80 \mathrm{mg}$ every 4 weeks [Q4W], -6.1) vs. placebo (-3.4) ( $p<0.001$, both comparisons) and higher rates of remission of depressive symptoms (ixekizumab Q2W, 45.2\%; ixekizumab Q4W, 33.6\%) vs. placebo (17.8\%) ( $p \leq 0.01$, both comparisons). Patients treated with ixekizumab also had significant reductions in hsCRP and PASI compared to placebo. Etanercept treatment was not associated with significant improvements in depressive symptoms compared to placebo. Conclusions: In this comorbid population, 12 weeks of ixekizumab therapy resulted in remission of depression for approximately $40 \%$ of patients and improved systemic inflammation as indicated by hsCRP.

(c) 2017 The Author(s)

Published by S. Karger AG, Basel

Christopher E.M. Griffiths is a National Institute for Health Research Senior Investigator.

Susan G. Ball, PhD

Lilly Corporate Center

240 E McCarty Street

Indianapolis, IN 46285 (USA)

E-Mail Ball_susan_g@ lilly.com 


\section{Introduction}

Depression is a common comorbidity in patients with plaque psoriasis. The prevalence rate of comorbid depression varies across studies, with a recent US population survey finding comorbid depressive illness in $16.5 \%$ of patients with psoriasis [1]. Within a general medical database, Kurd et al. [2] found that patients with psoriasis had a significantly greater risk of depression, anxiety, or suicidality (thoughts or behaviors) compared to matched controls, and the risk was associated with the severity of psoriasis. A meta-analysis found an overall prevalence of $12 \%$ for depressive illness and $28 \%$ for depressive symptoms among patients with psoriasis [3].

While the psychological burden of psoriasis may be a stressor that contributes to the development of depression [4], the increased risk of depression may also be due to shared physiological mechanisms [5]. Psoriasis is recognized as an immune-mediated disorder consisting of dysregulated cytokine networks that lead to chronic inflammation [6, 7]. Similarly, some studies have suggested an association between depression and increased levels of proinflammatory cytokines, such as tumor necrosis factor- $\alpha$ and interleukin (IL)-6 in the blood [8]. As one marker of systemic inflammation, high-sensitivity C-reactive protein (hsCRP) has been shown to be elevated in some patients with depression [9] and in patients with psoriasis [10].

Ixekizumab is a high-affinity monoclonal antibody that selectively targets the proinflammatory cytokine IL$17 \mathrm{~A}$ and has demonstrated efficacy for the treatment of moderate-to-severe plaque psoriasis (psoriasis) [11]. The objective of this analysis was to examine the effects of ixekizumab therapy on depressive symptoms and systemic inflammation in patients with psoriasis and comorbid depressive symptoms. This report focuses on the subgroup of patients who had at least moderately severe depressive symptoms in the pivotal phase 3 ixekizumab psoriasis trials.

\section{Methods}

\section{Study Design}

Data were integrated from the 12-week induction period of 3 pivotal, phase 3, double-blind, randomized, placebo-controlled trials of ixekizumab (UNCOVER-1, UNCOVER-2, and UNCOVER-3) for the treatment of patients with psoriasis. Patients were randomized to receiving either ixekizumab $80 \mathrm{mg}$ every 4 weeks (Q4W; $n=1,165)$, ixekizumab $80 \mathrm{mg}$ every 2 weeks (Q2W; $n=$ $1,169)$, or placebo $(n=792)$. For the ixekizumab treatment groups, patients received an initial dose of ixekizumab $160 \mathrm{mg}$. The induction periods of UNCOVER-2 and UNCOVER-3 also had the active comparator group of etanercept $50 \mathrm{mg}$ twice weekly $(n=739)$. Etanercept comparisons were made using integrated data from these 2 studies.

\section{Participants}

The patient subset for this report was derived from the intentto-treat (ITT) population of the trials. The comorbid depressive symptom population was defined as patients who had a 16-item Quick Inventory for Depressive Symptomatology - Self-Report (QIDS-SR 16 ) total score $\geq 11$ at baseline (at least moderately severe depression). Patients were adult (age $\geq 18$ years) outpatients who had been diagnosed with psoriasis for at least 6 months who met the following severity criteria: body surface area percentage coverage score $\geq 10 \%$, Psoriasis Area and Severity Index (PASI) score $\geq 12$, and static Physician Global Assessment score $\geq 3$ (at least moderate severity). Key exclusion criteria included: other forms of psoriasis, recent use of biological therapy within specific washout periods, an unstable serious medical disease, a recent serious infection, presence of a significant uncontrolled neuropsychiatric disorder, a history of suicide attempt or current suicide ideation (as determined by a score of 3 [frequent thoughts of suicide, a plan, or an attempt] on QIDS-SR 16 item 12 for thoughts of death/suicide or by investigator opinion), a history of malignant disease, and laboratory values outside of specific ranges. All patients gave written informed consent prior to study procedures. The studies were approved by institutional review boards and regulatory authorities and registered as UNCOVER-1, UNCOVER-2, and UNCOVER-3 with ClinicalTrials.gov (NCT01474512, NCT01597245, and NCT01646177, respectively).

\section{Measures}

Depressive symptoms were measured using the QIDS-SR ${ }_{16}$, a reliable and well-validated 16 -item self-report questionnaire that assesses 9 domains (sad mood, concentration, self-criticism, suicidal ideation, interest, energy/fatigue, sleep disturbance [insomnia or hypersomnia], appetite/weight disturbance [decrease or increase], and psychomotor agitation or retardation) [12-14]. The total score ranges from 0 to 27 ; individual scores are interpreted as 0-5 (none), 6-10 (mild severity), 11-15 (moderate severity), 16-20 (severe), and 21-27 (very severe) [13]. The QIDS-SR 16 was administered at baseline and week 12 .

Serum hsCRP was assessed at baseline through week 12 by immunonephelometry using a Siemens BN ${ }^{\mathrm{TM}}$ II nephelometer. Investigative sites were blinded to the results.

Psoriasis disease severity was measured by the PASI, which is a physician-rated instrument that combines assessment of the extent of body surface involvement in 4 anatomical regions (head, trunk, arms, and legs) and the severity of desquamation (scaling), erythema, and plaque induration/infiltration (thickness). PASI assessments yield an overall score of 0 (no psoriasis) to 72 (most severe disease) [15]. Improvements in the severity of psoriasis are reported as a percentage of the improvement in PASI compared to the baseline value; for example, a PASI of 75 indicates that the patient's postbaseline score is at least a $75 \%$ improvement compared to their baseline score. Complete resolution of psoriasis is defined as a $100 \%$ improvement in PASI score from baseline (PASI 100). The PASI assessment was administered at baseline and through week 12 .
Impact of Ixekizumab on Comorbid

Depression in Patients with Psoriasis
Psychother Psychosom 2017;86:260-267 DOI: $10.1159 / 000479163$ 
Table 1. Baseline demographics and illness characteristics of patients with moderate-to-severe plaque psoriasis and comorbid depressive symptoms

\begin{tabular}{lllll}
\hline & PBO $(n=93)$ & IXE Q4W $(n=120)$ & IXE Q2W $(n=107)$ & Total $(n=320)$ \\
\hline Age, years & $46.8 \pm 13.1$ & $44.2 \pm 13.3$ & $45.5 \pm 12.4$ & $45.4 \pm 13.0$ \\
Males & 57.0 & 55.8 & 57.9 & 56.9 \\
Whites & 88.2 & 87.5 & 87.9 & 87.8 \\
Weight, kg & $86.9 \pm 22.1$ & $92.6 \pm 27.5$ & $88.6 \pm 24.5$ & $89.6 \pm 25.0$ \\
Previous biological therapy & 36.6 & 26.7 & 32.7 & 31.6 \\
Duration of Ps, years & $18.6 \pm 13.1$ & $16.2 \pm 11.0$ & $17.1 \pm 12.5$ & $17.2 \pm 12.1$ \\
sPGA (severe, very severe $\left.{ }^{1}\right)$ & 53.8 & 60.8 & 49.5 & 55.0 \\
PASI & $22.1 \pm 9.0$ & $23.3 \pm 8.9$ & $22.1 \pm 8.8$ & $22.5 \pm 8.9$ \\
QIDS-SR ${ }_{16}$ score & $14.0 \pm 3.1$ & $14.2 \pm 2.6$ & $13.8 \pm 2.6$ & $14.0 \pm 2.8$ \\
Psoriatic arthritis & 39.8 & 32.5 & 42.1 & 37.8 \\
hsCRP, mg/L & $8.6 \pm 14.3$ & $7.6 \pm 12.1$ & $9.7 \pm 20.5$ & $8.6 \pm 15.9$ \\
hsCRP $(>5$ mg/L), $n / N x(\%)$ & $40 / 89(44.9)$ & $43 / 117(36.8)$ & $42 / 100(42.0)$ & $125 / 306(40.8)$
\end{tabular}

Values are presented as means \pm SD or percents unless otherwise stated. hsCRP, high sensitivity C-reactive protein; IXE Q2W, ixekizumab $80 \mathrm{mg}$ every 2 weeks; IXE Q4W, ixekizumab $80 \mathrm{mg}$ every 4 weeks; $n / \mathrm{Nx}=$ number of patients who met criteria/number of patients who had a baseline or postbaseline value; PBO, placebo; PASI, Psoriasis Area Severity Index; Ps, psoriasis; QIDS-SR ${ }_{16}$, 16-item Quick Inventory for Depressive Symptomatology - Self-Report; sPGA, static Physician Global Assessment. ${ }^{1}$ Severe baseline sPGA (score $\geq 4) .{ }^{2}$ Self-reported and not based on a formal medical diagnosis.

\section{Statistical Methods}

Placebo comparisons were made using integrated induction data from the 3 studies. Etanercept comparisons were made using integrated data from UNCOVER 2 and UNCOVER 3.

Unless otherwise specified, analyses were conducted on the comorbid depressive symptom population, which is a subset of the ITT population. The last observation carried forward (LOCF) method was used to account for missing data unless otherwise specified.

The LOCF mean changes from baseline to week 12 in the QIDS-SR 16 total score, 9 QIDS-SR ${ }_{16}$ domain scores, and hsCRP were analyzed using an analysis of covariance (ANCOVA) model with treatment, study, and baseline values as covariates. Improvements in QIDS-SR 16 item 12 refer to postbaseline values that are less than the reference baseline value.

Categorical data frequencies were summarized by treatment group and analyzed using the Cochran-Mantel-Haenszel test stratified by study. Clinically meaningful improvement in depressive symptoms (response) was defined as $\geq 50 \%$ improvement in the QIDS-SR 16 total score from baseline to week 12 . Remission of depressive symptoms was defined as a QIDS-SR ${ }_{16}$ total score $\leq 5$ at week 12. Elevations of hsCRP, defined as hsCRP $>5 \mathrm{mg} / \mathrm{L}$, were summarized at baseline, at week 12 , and by the presence or absence of psoriatic arthritis (PsA) at baseline.

Improvements in psoriasis as measured by the PASI (PASI 75, PASI 90, and PASI 100) from baseline to week 12 were analyzed using the Cochran-Mantel-Haenszel test stratified by study. If missing PASI data occurred, the patient was considered a nonresponder.

Categorical assessments of QIDS-SR 16 total scores and QIDS$\mathrm{SR}_{16}$ item 12 scores compared maximum postbaseline scores with maximum baseline scores.

The association between changes from baseline to week 12 (LOCF) was computed using a Pearson product correlation. The correlation of QIDS-SR 16 total scores and hsCRP values, and the correlation of PASI improvements $(<75,75$ to $<90,90$ to $<100$, and $100)$ and QIDS-SR 16 remission status, were summarized.

\section{Role of the Funding Source}

The studies were designed by Eli Lilly and Company (Lilly) while consulting with external experts. Data were collected by the investigators (the complete dataset was kept at the central processing database held by Lilly). The analyses were developed by the academic investigators who had access to the data. The academic investigators made decisions about submission for publication in collaboration with Lilly, but all authors, including those employed by Lilly, had to approve the document prior to submission for publication.

\section{Results}

Within the placebo-controlled integrated dataset across the 3 studies $(n=3,126)$, approximately $10 \%$ of the patients met the criterion of a baseline QIDS-SR 16 total score $\geq 11$ (placebo, $n=93$; ixekizumab $80 \mathrm{mg}$ every 4 weeks [Q4W], $n=120$; and ixekizumab $80 \mathrm{mg}$ every 2 weeks [Q2W], $n=107$; Table 1). The demographics and baseline illness characteristics of the comorbid depressive symptom population were similar to those of the overall ITT population, with the exception of the percent of patients who had comorbid PsA ( $\sim 40 \%$ in the comorbid depressive population vs. $24 \%$ in the overall ITT population). Within the analysis set that includes etanercept 


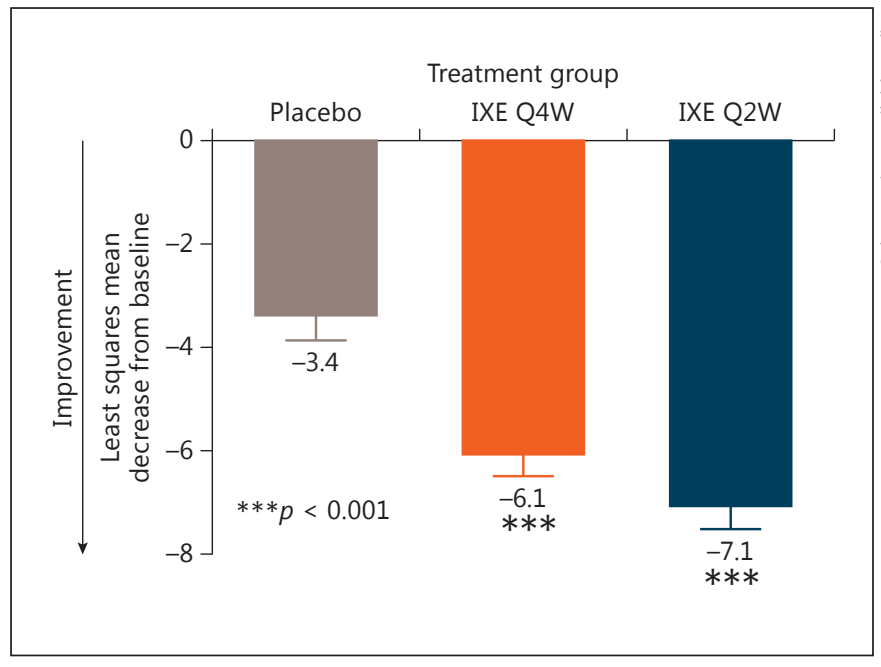

Fig. 1. Least squares mean change in QIDS-SR 16 total score from baseline at week 12 (last observation carried forward) by treatment group. Values are presented as least squares means \pm standard error. IXE Q2W, ixekizumab 80 mg every 2 weeks; IXE Q4W, ixekizumab 80 mg every 4 weeks; QIDS-SR 16,16 -item Quick Inventory for Depressive Symptomatology - Self-Report.

( $n=2,562)$, approximately $10 \%$ of patients had a baseline QIDS-SR 16 total score $\geq 11$ (placebo, $n=47$; etanercept, $n=66$; ixekizumab Q4W, $n=74$; and ixekizumab Q2W, $n=68)$.

\section{Effect of Treatment on Depressive Symptoms}

After 12 weeks, patients with comorbid depressive symptoms who were treated with ixekizumab Q4W or Q2W had significantly greater mean reductions in their QIDS-SR 16 total score compared to patients who received placebo (ixekizumab Q4W, $-6.1 \pm 0.41$; ixekizumab Q2W, $-7.1 \pm 0.44$; and placebo, $-3.4 \pm 0.48$; $p<0.001$, both comparisons; Fig. 1). The proportion of patients who met the criteria for clinical response in depressive symptoms was also significantly greater in patients treated with ixekizumab Q4W $(46.6 \%, p<0.01)$ and ixekizumab Q2W $(61.5 \%, p<0.001)$ compared to placebo $(25.6 \%)$ at week 12 . Similarly, a significantly greater proportion of patients in both ixekizumab treatment groups (Q4W, 33.6\%, $p=0.01$; Q2W, 45.2\%, $p<0.001)$ met criteria for remission of depressive symptoms at week 12 compared to patients who received placebo (17.8\%; Fig. 2). Within the 2 studies that included etanercept, patients treated with etanercept had numerically higher values but did not differ significantly from patients treated with placebo in terms of their mean change from baseline

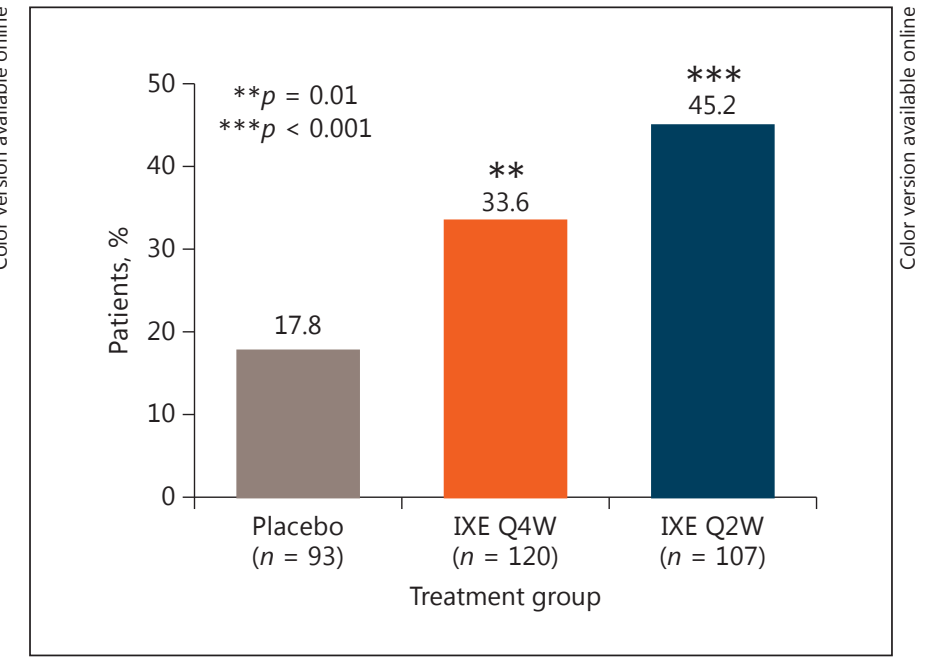

Fig. 2. Proportion of patients who met remission criteria for depressive symptoms (QIDS-SR 16 total score $\leq 5$ at week 12 [last observation carried forward]) by treatment group. IXE Q2W, ixekizumab 80 mg every 2 weeks; IXE Q4W, ixekizumab 80 mg every 4 weeks; QIDS-SR 16 , 16-item Quick Inventory for Depressive Symptomatology - Self-Report.

for QIDS-SR 16 total score $(-4.1 \pm 0.57$ vs. $-3.7 \pm 0.68$, respectively) and the rate of clinical response for depression (30.2 vs. $26.7 \%$, respectively) or in remission of depression (22.2 vs. $13.3 \%$, respectively).

There were significant improvements at week 12 in patients treated with either dose of ixekizumab compared to placebo in the following QIDS-SR ${ }_{16}$ domains: sad mood, self-criticism, suicidal ideation (with the exception of the ixekizumab Q2W group), interest, energy/fatigue, and agitation/retardation. Of particular clinical importance, a numerically higher proportion of patients from the combined ixekizumab treatment groups showed improvement from baseline on item 12 (thoughts of death or suicide) compared to patients treated with placebo (22.2 vs. $15.6 \%)$, whereas a numerically higher proportion of placebo-treated patients had a worsening from baseline on this item compared to patients from the pooled ixekizumab group (6.7 vs. $3.6 \%)$.

\section{Effect of Treatment on Systemic Inflammation}

At baseline, patients with comorbid depressive symptoms had higher mean and median serum hsCRP levels (8.57 and $4.02 \mathrm{mg} / \mathrm{L}$, respectively) relative to patients from the overall ITT population of the phase 3 pivotal trials (5.89 and $2.95 \mathrm{mg} / \mathrm{L}$, respectively). Approximately $41 \%$ of the patients with comorbid depressive symptoms met the
Impact of Ixekizumab on Comorbid Depression in Patients with Psoriasis
Psychother Psychosom 2017;86:260-267 DOI: $10.1159 / 000479163$ 


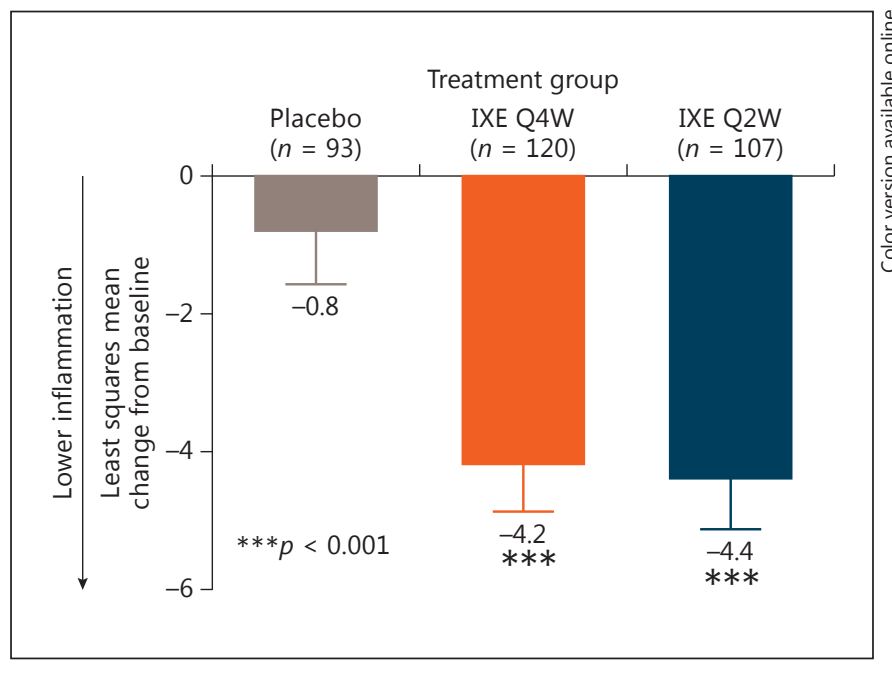

Fig. 3. Least squares mean change from baseline in hsCRP (mg/L) at week 12 (last observation carried forward) by treatment group. Values are presented as least squares means \pm standard error. hsCRP, high-sensitivity C-reactive protein; IXE Q2W, ixekizumab $80 \mathrm{mg}$ every 2 weeks; IXE Q4W, ixekizumab $80 \mathrm{mg}$ every 4 weeks.

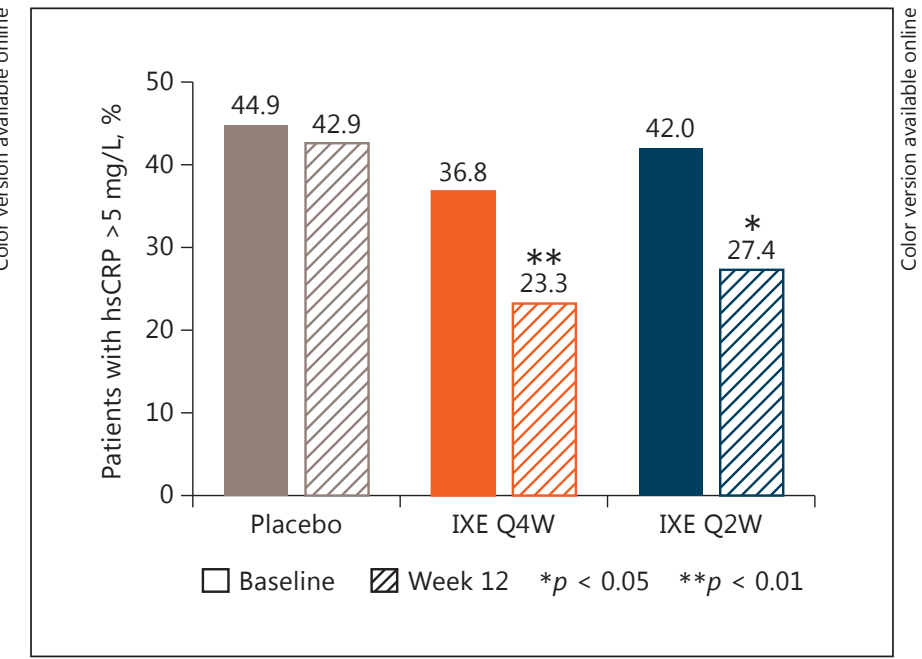

Fig. 4. Percentage of patients with at least moderately severe depressive symptoms who met criteria for elevated hsCRP at baseline and at week 12 (last observation carried forward) by treatment group. hsCRP, high-sensitivity C-reactive protein; IXE Q2W, ixekizumab 80 mg every 2 weeks; IXE Q4W, ixekizumab 80 mg every 4 weeks. criteria for elevated hsCRP ( $>5 \mathrm{mg} / \mathrm{L})$ at baseline. Patients with comorbid depressive symptoms who were treated with ixekizumab had significantly greater mean reductions in hsCRP at week $12(\mathrm{Q} 4 \mathrm{~W},-4.2 \pm 0.67 \mathrm{mg} / \mathrm{L} ; \mathrm{Q} 2 \mathrm{~W}$, $-4.4 \pm 0.72 \mathrm{mg} / \mathrm{L})$ compared to placebo $(-0.8 \pm 0.77 \mathrm{mg} / \mathrm{L})$ ( $p<0.001$, both comparisons) (Fig. 3). Similarly, the median change in hsCRP was $-0.73 \mathrm{mg} / \mathrm{L}$ (IQR -3.09 to 0.30 ) and $-0.62 \mathrm{mg} / \mathrm{L}$ (IQR -3.5 to 0.15 ) for ixekizumab Q4W and Q2W, respectively, and it was $+0.20 \mathrm{mg} / \mathrm{L}$ (IQR -1.8 to 1.1) for placebo. After 12 weeks, compared to the baseline frequency, the proportion of patients in the ixekizumab treatment groups with elevated hsCRP had decreased by approximately a third (35-37\%) and it was significantly different from that for placebo (Fig. 4). The Pearson product correlation between mean reduction in hsCRP value and mean improvement in QIDS-SR ${ }_{16}$ total score from the comorbid depressive symptom population was $r=0.11(p=0.001)$. Patients with comorbid depressive symptoms who were treated with etanercept also had a significant mean reduction in hsCRP $(-3.2 \pm 1.04 \mathrm{mg} / \mathrm{L})$ compared to their placebo counterparts $(+0.12 \pm 1.2$ $\mathrm{mg} / \mathrm{L})$ after 12 weeks $(p=0.033)$. The median change was $-1.34 \mathrm{mg} / \mathrm{L}$ (IQR -2.7 to 0.16 ) for etanercept and +0.25 $\mathrm{mg} / \mathrm{L}$ (IQR -1.4 to 1.3 ) for placebo.

The reduction in hsCRP was also examined by the presence or absence of PsA at baseline in the subgroup of patients with comorbid depressive symptoms. A greater proportion of patients with comorbid PsA met criteria for elevated hsCRP at baseline compared to patients who did not have comorbid PsA (47.0 and 37.2\%, respectively). After 12 weeks, the proportion of patients with elevated hsCRP who were treated with ixekizumab decreased by a similar percentage between the subgroups of patients with or without PsA (data not shown).

\section{Effect of Treatment on Skin Clearance}

Similar to the overall ITT population $[11,16]$, patients with comorbid depressive symptoms who were treated with ixekizumab had significantly greater improvement in PASI scores from baseline compared to placebo $(p<$ 0.001 , all comparisons). The proportion of patients who achieved a PASI 75 at week 12 was $78.3 \%$ (ixekizumab Q4W), 86.0\% (ixekizumab Q2W), and 1.1\% (placebo). The proportion of patients who had nearly complete resolution (PASI 90) was 60.0\% (ixekizumab Q4W), 68.2\% (ixekizumab Q2W), and 0\% (placebo). The response rates for complete resolution of psoriasis (PASI 100) were 31.7, 37.4, and 0\% for ixekizumab Q4W, ixekizumab Q2W, and placebo, respectively. The Pearson correlation between PASI improvement scores and depression remission status at week 12 for the comorbid depressive population was $r=-0.25$. 


\section{Discussion}

From the integrated database, approximately $10 \%$ of patients with psoriasis had at least moderately severe depressive symptoms as defined by a QIDS-SR 16 total score $\geq 11$ at baseline. Patients treated with ixekizumab therapy had significant mean reductions from baseline in depressive symptoms and systemic inflammation, as represented by circulating levels of serum hsCRP. Within this comorbid population, $45 \%$ of patients treated with the recommended dose of ixekizumab (80 mg Q2W) over the 12-week induction period achieved remission of their depressive symptoms. The proportion of patients who achieved PASI 75, PASI 90, or PASI 100 from this subgroup at week 12 was comparable to the rates observed within the individual phase 3 trials after 12 weeks [11]. The majority of patients achieved either meaningful improvement or nearly complete resolution of their psoriatic plaques.

The effect of ixekizumab on depressive symptoms is consistent with results from other biological therapies that have shown significant reductions in depressive symptoms in patients with psoriasis [17]. However, the findings from the etanercept treatment group differ from a published report in which the percentage of patients with psoriasis treated with etanercept who had a $50 \%$ improvement in depressive symptoms was significantly higher in patients treated with placebo (43 vs. $32 \%$ ) [18]. In contrast, the findings from the current study showed that etanercept-treated patients did not have a significant improvement in depressive symptoms compared to placebo-treated patients. The difference in outcomes between these studies may be related to differences in the severity symptoms of the study population and the assessment tool for depression. Only $2 \%$ of the sample from Tyring et al. [18] met criteria for at least moderately severe depressive symptoms at baseline, and depressive symptoms were assessed by the Hamilton Depression Rating Scale, a clinician-rated measure [19].

The difference in outcomes between etanercept and ixekizumab relative to placebo may also be potentially related to the underlying pathophysiology given the difference in mechanisms of action of etanercept, a tumor necrosis factor- $\alpha$ inhibitor, and ixekizumab, an IL-17A antagonist. Although, both etanercept and ixekizumab resulted in anti-inflammatory effects as measured by hsCRP levels, the lack of an effect on depressive symptoms by etanercept in the present study suggests that tumor necrosis factor- $\alpha$ may not play a primary role in the cytokine dysregulation underlying the comorbidity between psoriasis and depressive symptoms. The literature suggests that the IL-17A pathways may have potential CNS effects [20] in which the cytokine IL-6 plays a specific role in increased immune-inflammation through the regulation of Th17 cells and IL-17 production [21].

The improvement in depressive symptoms following ixekizumab therapy occurred across 7 of the 9 symptom domains associated with a major depressive disorder. Notably, given that patients with psoriasis are at an increased risk for suicide-related thoughts and behaviors [2], patients treated with ixekizumab did not experience significant changes in thoughts of death or suicide, and numerically ixekizumab therapy resulted in greater improvement on this item compared to placebo. Interpretation of this finding must consider that patients with frequent suicidal ideation (that is, a score of 3 on item 12 of the QIDS$\mathrm{SR}_{16}$ ) were excluded at baseline. Nonetheless, patients with comorbid depressive symptoms did not experience a significant increase in suicide-related thoughts and behaviors with ixekizumab therapy. The finding of no increased risk of worsening of depression or suicide in the comorbid psoriasis and depressive symptoms is consistent with analyses for the overall ITT patient population $[22,23]$.

A strength of this analysis is that the ixekizumab psoriasis program included over 3,000 patients, which allowed identification of over $300(\sim 10 \%)$ patients with at least moderately severe symptoms. In addition, depressive and systemic inflammatory outcomes were assessed using valid and reliable measures. Limitations of the analyses are that this study was a post hoc analysis of a subgroup from an integrated dataset; patients were not systematically diagnosed for psychiatric illness, and depressive symptoms were measured only at baseline and at week 12, which precludes time course analyses.

In summary, in patients with moderate-to-severe psoriasis and clinically relevant depressive symptoms, 12 weeks of therapy with ixekizumab was superior to placebo in improving depressive symptoms, reducing the inflammatory biomarker of hsCRP, and attaining high levels of skin clearance.

\section{Acknowledgments}

We thank Chad D. Walls, PhD, and Brian S. Comer, PhD, both full-time employees of Eli Lilly and Company, for writing and editorial assistance.

This study was supported by Eli Lilly and Company.

Psychother Psychosom 2017;86:260-267

DOI: $10.1159 / 000479163$ 


\section{Disclosure Statement}

All authors and representatives of Eli Lilly and Company reviewed and approved the manuscript. The authors maintained control over the final content.

Christopher E.M. Griffiths reports grants and personal fees from Eli Lilly during the conduction of this study, grants and personal fees from AbbVie, Janssen, Celgene, Novartis, Pfizer, and GSK-Stiefel, grants from Sandoz, LEO Pharma, MMS, MSD, Sanofi, and Roche, personal fees from Amgen, UCB Pharma, Sun Pharmaceuticals, and MedScape, and stock/stock options from CG Skin outside of the submitted work.

M. Fava is an advisory board member/consultant with lifetime disclosure for Abbott Laboratories, Acadia, Affectis Pharmaceuticals AG, Alkermes Inc., Amarin Pharma Inc., Aspect Medical Systems, AstraZeneca, Auspex Pharmaceuticals, Avanir Pharmaceuticals, AXSOME Therapeutics, Bayer AG, Best Practice Project Management Inc., Biogen, BioMarin Pharmaceuticals Inc., Biovail Corporation, BrainCells Inc., Bristol-Myers Squibb, CeNeRx BioPharma, Cephalon Inc., Cerecor, CNS Response Inc., Compellis Pharmaceuticals, Cypress Pharmaceutical Inc., DiagnoSearch Life Sciences Pvt. Ltd., Dinippon Sumitomo Pharma Co. Inc., Dov Pharmaceuticals Inc., Edgemont Pharmaceuticals Inc., Eisai Inc., Eli Lilly and Company, EnVivo Pharmaceuticals Inc., ePharmaSolutions, EPIX Pharmaceuticals Inc., Euthymics Bioscience Inc., Fabre-Kramer Pharmaceuticals Inc., Forest Pharmaceuticals Inc., Forum Pharmaceuticals, GenOmind LLC, GlaxoSmithKline, Grunenthal $\mathrm{GmbH}$, i3 Innovus/Ingenis, Intracellular, Janssen Phar- maceutica, Jazz Pharmaceuticals Inc., Johnson \& Johnson Pharmaceutical Research \& Development LLC, Knoll Pharmaceuticals Corp., Labopharm Inc., Lorex Pharmaceuticals, Lundbeck Inc., MedAvante Inc., Merck \& Co. Inc., MSI Methylation Sciences Inc., Naurex Inc., Nestle Health Sciences, Neuralstem Inc., Neuronetics Inc., NextWave Pharmaceuticals, Novartis AG, Nutrition 21, Orexigen Therapeutics Inc., Organon Pharmaceuticals, Osmotica, Otsuka Pharmaceuticals, Pamlab LLC, Pfizer Inc., PharmaStar, Pharmavite ${ }^{\circledR}$ LLC, PharmoRx Therapeutics, Precision Human Biolaboratory, Prexa Pharmaceuticals Inc., PPD, Puretech Ventures, PsychoGenics, Psylin Neurosciences Inc., RCT Logic LLC (formerly Clinical Trials Solutions LLC), Rexahn Pharmaceuticals Inc., Ridge Diagnostics Inc., Roche, Sanofi-Aventis US LLC, Sepracor Inc., Servier Laboratories, Schering-Plough Corporation, Solvay Pharmaceuticals Inc., Somaxon Pharmaceuticals Inc., Somerset Pharmaceuticals Inc., Sunovion Pharmaceuticals, Supernus Pharmaceuticals Inc., Synthelabo, Taisho Pharmaceutical, Takeda Pharmaceutical Company Limited, Tal Medical Inc., Tetragenex Pharmaceuticals Inc., TransForm Pharmaceuticals Inc., Transcept Pharmaceuticals Inc., Vanda Pharmaceuticals Inc., and VistaGen. No stock/other financial options have been received to date. Equity Holdings: Compellis; PsyBrain, Inc.

A.H. Miller and M.H. Rapaport report no disclosure of conflicts of interests.

J. Russell is a retiree of Eli Lilly and Company and owns stock. S. Ball, W. Xu, and N. Acharya are full-time employees of Eli Lilly and Company and own stock.

\section{References}

1 Cohen BE, Martires KJ, Ho RS: Psoriasis and the risk of depression in the US population: National Health and Nutrition Examination Survey 2009-2012. JAMA Dermatol 2016; 152:73-79.

2 Kurd SK, Troxel AB, Crits-Christoph P, Gelfand JM: The risk of depression, anxiety, and suicidality in patients with psoriasis: a population-based cohort study. Arch Dermatol 2010;146:891-895.

3 Dowlatshahi EA, Wakkee M, Arends LR, Nijsten T: The prevalence and odds of depressive symptoms and clinical depression in psoriasis patients: a systematic review and meta-analysis. J Invest Dermatol 2014;134:1542-1551.

4 Dalgard FJ, Gieler U, Tomas-Aragones L, Lien L, Poot F, Jemec GB, Misery L, Szabo C, Linder D, Sampogna F, Evers AW, Halvorsen JA, Balieva F, Szepietowski J, Romanov D, Marron SE, Altunay IK, Finlay AY, Salek SS, Kupfer J: The psychological burden of skin diseases: a cross-sectional multicenter study among dermatological out-patients in $13 \mathrm{Eu}$ ropean countries. J Invest Dermatol 2015;135: 984-991.

5 Connor CJ, Liu V, Fiedorowicz JG: Exploring the physiological link between psoriasis and mood disorders. Dermatol Res Pract 2015; 2015:409637.
6 Nickoloff BJ, Xin H, Nestle FO, Qin JZ: The cytokine and chemokine network in psoriasis. Clin Dermatol 2007;25:568-573.

7 Lowes MA, Suarez-Farinas M, Krueger JG: Immunology of psoriasis. Annu Rev Immunol 2014;32:227-255.

8 Dowlati Y, Herrmann N, Swardfager W, Liu H, Sham L, Reim EK, Lanctot KL: A metaanalysis of cytokines in major depression. Biol Psychiatry 2010;67:446-457.

9 Wium-Andersen MK, Orsted DD, Nielsen SF, Nordestgaard BG: Elevated C-reactive protein levels, psychological distress, and depression in 73,131 individuals. JAMA Psychiatry 2013;70:176-184.

10 Beygi S, Lajevardi V, Abedini R: C-reactive protein in psoriasis: a review of the literature. J Eur Acad Dermatol Venereol 2014;28:700711.

11 Griffiths CE, Reich K, Lebwohl M, van de Kerkhof P, Paul C, Menter A, Cameron GS, Erickson J, Zhang L, Secrest RJ, Ball S, Braun DK, Osuntokun OO, Heffernan MP, Nickoloff BJ, Papp K, UNCOVER-2 and UNCOVER-3 investigators: Comparison of ixekizumab with etanercept or placebo in moderate-to-severe psoriasis (UNCOVER-2 and UNCOVER-3): results from two phase 3 randomised trials. Lancet 2015;386: 541-551.
12 Trivedi MH, Rush AJ, Ibrahim HM, Carmody TJ, Biggs MM, Suppes T, Crismon ML, Shores-Wilson K, Toprac MG, Dennehy EB, Witte B, Kashner TM: The inventory of depressive symptomatology, clinician rating (IDS-C) and self-report (IDS-SR), and the quick inventory of depressive symptomatology, clinician rating (QIDS-C) and self-report (QIDS-SR) in public sector patients with mood disorders: a psychometric evaluation. Psychol Med 2004;34:73-82.

13 Quick inventory of depression symptomology. http://www.ids-qids.org/ (accessed May 12, 2016)

14 Reilly TJ, MacGillivray SA, Reid IC, Cameron IM: Psychometric properties of the 16-item quick inventory of depressive symptomatology: a systematic review and meta-analysis. J Psychiatr Res 2015;60:132-140.

15 Fredriksson T, Pettersson U: Severe psoriasis - oral therapy with a new retinoid. Dermatologica 1978;157:238-244.

16 Gordon KB, Blauvelt A, Papp KA, Langley RG, Luger T, Ohtsuki M, Reich K, Amato D, Ball SG, Braun DK, Cameron GS, Erickson J, Konrad RJ, Muram TM, Nickoloff BJ, Osuntokun OO, Secrest RJ, Zhao F, Mallbris L, Leonardi CL: Phase 3 trials of ixekizumab in moderate-to-severe plaque psoriasis. N Engl J Med 2016;375:345-356. 
17 Fleming P, Roubille C, Richer V, Starnino T, McCourt C, McFarlane A, Siu S, Kraft J, Lynde C, Pope JE, Keeling S, Dutz J, Bessette L, Bissonnette R, Haraoui B, Gulliver WP: Effect of biologics on depressive symptoms in patients with psoriasis: a systematic review. J Eur Acad Dermatol Venereol 2015;29:10631070.

18 Tyring S, Gottlieb A, Papp K, Gordon K, Leonardi C, Wang A, Lalla D, Woolley $\mathrm{M}$, Jahreis A, Zitnik R, Cella D, Krishnan R: Etanercept and clinical outcomes, fatigue, and depression in psoriasis: double-blind placebocontrolled randomised phase III trial. Lancet 2006;367:29-35.
19 Hamilton Rating Scale for Depression (HAMD). http://www.serene.me.uk (accessed May 12, 2016)

20 Waisman A, Hauptmann J, Regen T: The role of IL-17 in CNS diseases. Acta Neuropathol 2015;129:625-637.

21 Anderson G, Kubera M, Duda W, Lason W, Berk M, Maes M: Increased IL-6 trans-signaling in depression: focus on the tryptophan catabolite pathway, melatonin and neuroprogression. Pharmacol Rep 2013;65:1647-1654.
22 Griffiths CE, Papp KA, Ball SG, Nickoloff BJ: Ixekizumab for psoriasis - authors' reply. Lancet 2016;387:226-227.

23 Strober B, Leonardi C, Papp KA, Mrowietz U, Ohtsuki M, Bissonnette R, Ferris LK, Paul C, Lebwohl M, Braun DK, Mallbris L, Wilhelm $\mathrm{S}, \mathrm{Xu} \mathrm{W}$, Ljungberg A, Acharya N, Reich K: Long-term safety profile of ixekizumab, an anti-interleukin-17A monoclonal antibody, in patients with moderate-to-severe psoriasis: an integrated analysis from 7 clinical trials. J Am Acad Dermatol 2017;76:432-440. 